\title{
Electrovariable gold nanoparticle films at liquid-liquid interfaces: from redox electrocatalysis to Marangoni-shutters
}

\author{
Grégoire C. Gschwend, $\uparrow$ Evgeny Smirnov, $\uparrow$ Pekka Peljo \\ and Hubert H. Girault*
}

Received 21st November 2016, Accepted 24th November 2016

DOI: $10.1039 / c 6 f d 00238 b$

Control over the physical properties of nanoparticle assemblies at a liquid-liquid interface is a key technological advancement to realize the dream of smart electrovariable nanosystems. Electrified interfaces, such as the interface between two immiscible electrolytes solutions (ITIES), are almost an ideal platform for realizing this dream. Here, we show that the Galvani potential difference across soft interfaces can be effectively used to manipulate: (i) the reactivity of gold nanoparticle assemblies through varying the Fermi level (both chemically and electrochemically); (ii) the location distribution of the nanoparticles at the liquid-liquid interface. In the first case, in addition to our previous studies on electron transfer reactions (ET) across the ITIES, we used intensity modulated photocurrent spectroscopy (IMPS) to study the kinetics of photo-induced electrochemical reactions at the ITIES. As expected, the direct adsorption of gold nanoparticles at the interface modifies the kinetics of the ET reaction (socalled, interfacial redox electrocatalysis), however it did not lead to an increased photocurrent by "plasmonic enhancement". Rather, we found that the product separation depends on double layer effects while the product recombination is controlled by the Galvani potential difference between the two phases. In the second case, we demonstrated that polarizing the ITIES caused migration of gold nanoparticles from the middle region of the cell to its periphery. We called such systems "Marangoni-type shutters". This type of electrovariable plasmonic system did not experience diffusion limitation in terms of the adsorption/ desorption of nanoparticles and the entire movement of nanoparticle assemblies happened almost instantly (within a second). It opens a fresh view on electrovariable plasmonics and presents new opportunities to create smart nanosystems at the ITIES driven with an electric field.

\section{Introduction}

The interface between two immiscible liquids, i.e. oil and water, is an extremely attractive scaffold for the self-assembly of nanoparticle (NP) films. ${ }^{1-3}$ Such 
interfaces have several advantages in comparison to solid-liquid interfaces: their pristine defect-free nature and mechanical pliability combined with self-healing capability and transparency. ${ }^{4-7}$

An interface between two immiscible electrolyte solutions (ITIES) provides another degree of freedom - application of the electric field across the interface. Thus, it makes it possible to create and/or to manipulate nanoparticle assemblies at soft interfaces. ${ }^{3,8-12}$ The main advantage of this is the capability to fabricate "smart" electrovariable nanosystems. ${ }^{8-10}$ However, despite enormous efforts and theoretical achievements in this field, to-date a working prototype of such a "smart" system has not been presented.

On the one hand, the physical properties of nanoparticle assemblies are affected by the electric field. For example, the Fermi level of nanoparticle assemblies can be tuned and manipulated with an external electric field ${ }^{13-16}$ to perform various electrochemical and electron transfer reactions. ${ }^{17,18}$ On the other hand, the physical position of the nanoparticles at the ITIES can be controlled by the electric field, anticipating the fabrication of "smart" plasmonic mirrors and filters in the near future. ${ }^{7,8,10}$ Therefore, to set the canvas of the current article, we shall present here the recent progress in both of the above mentioned topics.

As suggested by Samec et al. ${ }^{19,20}$ in the 1980s, the ET reactions at an ITIES were thought to be truly heterogeneous (HET). For truly HET reactions, redox couples namely, $\mathrm{D}^{+} / \mathrm{D}^{0}$ in one phase and $\mathrm{A}^{0} / \mathrm{A}^{-}$in the other phase - were supposed to react only at the interface where electron transfer reactions occurred. Later, Kihara et al.,$^{21}$ Osakai et al. ${ }^{22}$ and Katano et al. ${ }^{23}$ postulated that one of the reactants could partition to the adjacent phase and the ET reactions could occur in a single phase. Thus, it opened up a new homogenous ET mechanism with associated ion transfer (IT) reactions (so called, ET-IT mechanism). However, in several recent papers $^{14,17}$ we extensively showed both experimentally and with computer modelling that ET reactions occurring at the ITIES took place in two steps, where the first reaction was potential independent. The first step can be (i) the partition of one reactant into the adjunct phase, (ii) a truly heterogeneous ET reaction at the interface with the charge transfer coefficient close to 0 , or a combination of (i) and (ii). Thus, the potential dependence of the ET reaction stems either from the concomitant ion transfer reaction, or a truly heterogeneous back electron transfer reaction at the interface with the charge transfer coefficient close to 1 , or a mixture of both. ${ }^{17}$

Furthermore, we developed an in-depth theoretical explanation of interfacial redox electrocatalysis to describe the ET reaction at a gold NP functionalized ITIES. ${ }^{14,17}$ Additionally, other floating conductive electrocatalysts like carbon nanotubes, ${ }^{15}$ graphene-derivatives, ${ }^{24,26}$ precious metal nanoparticles ${ }^{14,25}$ etc. have been used in the redox electrocatalysis of, for example, oxygen reduction, ${ }^{\mathbf{1 8 , 2 6}}$ hydrogen evolution ${ }^{25,27,28}$ and metal deposition at the ITIES. ${ }^{24,29,30}$

Among other methods to study the ET phenomena at the ITIES, such as scanning electrochemical microscopy (SECM) ${ }^{31}$ an electron-conductor separated oil-water system (ECSOW) ${ }^{32}$ or in situ fluorescence spectroscopy, ${ }^{33}$ Fermin et al. ${ }^{34}$ and Samec et al. ${ }^{35}$ implemented intensity modulated photocurrent spectroscopy (IMPS) to investigate the photo-induced electron transfer reactions at the ITIES. This technique has the advantage of determining several parameters of the ET reactions at the ITIES, assuming a proper heterogeneous electron transfer model. The systems studied involved aqueous soluble zinc porphyrins (ZnTPPC, 
ZnTMPyP or ZnTPPS) ${ }^{36}$ as photoredox species and ferrocene- and quinonederivatives as electron donors or acceptors, respectively. While a clear potentialdependence of the rate constants was observed, the study was limited by equipment shortcomings and, thus, low data quality. Furthermore, in later works, plasmonic effects were claimed to be the main reason for the photocurrent enhancement once nanoparticles were added at the ITIES. ${ }^{37}$ In this earlier work of our group, Schaming et al. studied with IMPS the effect of a gold nanoparticle film adsorbed between the electron donor in the organic phase and the electron acceptor in the aqueous phase. The rationale behind this experiment was the "enhancement of the excitation" of the photoredox species, ZnTPPC, through "plasmonic effects" of the nanoparticles. Despite the fact that a larger photocurrent and changes in the reaction kinetics were observed, the real reason behind these observations was not clear. In particular, the film formation method required the injection of large amounts of methanol (of the order of $30 \%$ of the aqueous phase) as well as vigorous shaking of the biphasic system, which could significantly affect the final results, for example, by mixing the donor, acceptor and nanoparticles together.

Thus, one of the goals of the present work is to revisit the IMPS methodology and the possibility of the plasmonic effects to enhance the photocurrent with our current understanding of ET reactions at the ITIES, as well as the influence of the electric field on it. In this article we implement the model described in ref. 34 . Nevertheless, its relevance to describe the observed phenomena is still an open question.

Besides their reactivity, the physical distribution of the nanoparticles at a soft interface can be controlled with an external electric filed. Previously, Flatte, Kornyshev and Urbakh progressively developed the theory of nanoparticle "landing" at the ITIES from the bulk phase with an electric field to form mirrortype films. ${ }^{8-10}$ However, the most important limitation of its practical implementation was the very large diffusion time of the nanoparticles from the bulk to the interface, of the order of tens of seconds.

Using the ability to move nanoparticles in the horizontal direction of a flat soft interface can be a better solution for making electrovariable optical shutter-type elements than the above described approach of vertical landing at the polarized ITIES. Therefore, the implementation of the well-known (but still poorly understood) Marangoni effect to manipulate the position of nanoparticles at the ITIES is an attractive area of smart-optics development.

By definition, the Marangoni effect is the mass transfer along an interface between two fluids, liquid-liquid or liquid-gas, due to changes in the interfacial surface tension. In a classical theory developed by V. G. Levich ${ }^{38}$ the concept of capillary motion was derived in the presence of a surface tension imbalance on the free surface of a liquid-liquid interface. For example, Zhao and Pumera recently showed the implementation of the interlayer Marangoni effect to power the movements of a capsule at a liquid-liquid interface (LLI).$^{39}$ They observed that a macroscopic capsule moved due to the constant release of $N, N^{\prime}$-dimethylformamide (DMF) through a permeable membrane creating an interfacial surface tension gradient.

Recently, Scanlon et al. reported that carbon nanotubes supported at the ITIES were able to move at the interface from one side of the cell to another following an alternating electric field. ${ }^{28}$ 
Therefore, the second goal of this paper is to introduce a novel concept of electrovariable Marangoni-type shutters based on gold nanoparticles at the ITIES, as well as to highlight their rapid response to an external electric field.

\section{Results and discussion}

\section{Intensity modulated photocurrent spectroscopy (IMPS)}

We significantly improved an experimental procedure described by Schaming et $a l .{ }^{37}$ to clarify observations that they had made previously. First of all, we used a microinjection method ${ }^{14}$ to make a gold nanoparticle film prior to the addition of a porphyrin solution. This resulted in a clean organic phase|film|aqueous phase system without mixing the phases or the injection of large amounts of methanol. Secondly, a zwitterionic porphyrin, i.e. charged but with an overall neutrality, was selected instead of ZnTPPC, which has the tendency to aggregate upon small variations in the $\mathrm{pH}$. Furthermore, using both a neutral electron donor and a neutral electron acceptor solved the problem of concentration effects due to the polarization of the ITIES. Finally, the IMPS measurements were carried out with a completely automated and integrated setup that prevented artefacts and greatly improved the reproducibility of the experiments.

Fig. 1 shows a comparison of the Nyquist plots for the photocurrent $\left(j_{\text {photo }}\right)$ with and without gold nanoparticles recorded at the same Galvani potential difference $\Delta_{\mathrm{o}}^{\mathrm{w}} \phi=250 \mathrm{mV}$. The data were recorded at frequencies ranging from $30 \mathrm{~Hz}$ to $0.1 \mathrm{~Hz}$ for the cell composition described in Scheme 2 in the Experimental section. In the model of the heterogeneous electron transfer reaction developed by Fermin et al., ${ }^{34}$ real and imaginary parts of the photocurrent response are described with the following system of equations:

$$
j_{\text {photo }}^{\mathrm{Re}}=g \frac{k_{\mathrm{ps}}\left(k_{\mathrm{ps}}+k_{\mathrm{rec}}\right)-\omega^{2}\left(k_{\mathrm{rec}} R C+1\right)}{\left(k_{\mathrm{ps}}+k_{\mathrm{rec}}-\omega^{2} R C\right)+\left(\left(k_{\mathrm{ps}}+k_{\mathrm{rec}}+\frac{1}{R C}\right) \omega R C\right)^{2}}
$$

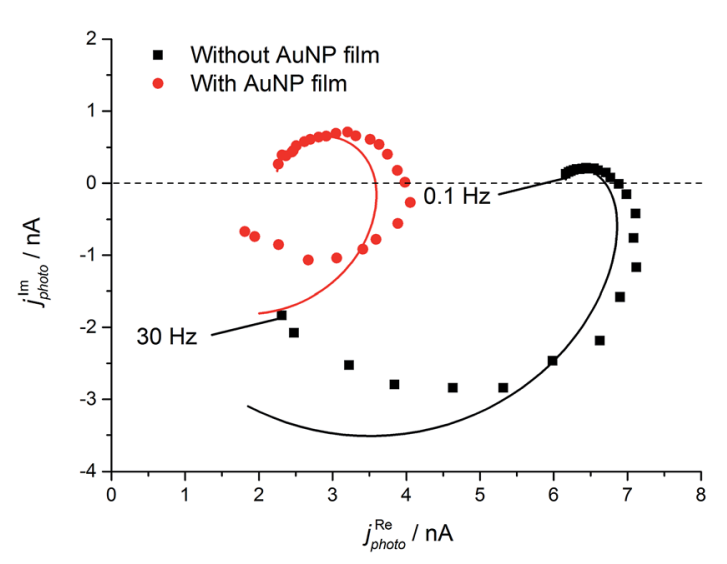

Fig. 1 Comparison of Nyquist plots recorded at $\Delta_{\circ}^{\mathrm{w}} \phi=250 \mathrm{mV}$ in the absence (black) and presence (red) of a gold nanoparticle film. Solid lines depict curve fittings in accordance with the model in ref. 34 and given by eqn (1) and (2). 


$$
j_{\text {photo }}^{\mathrm{Im}}=g \frac{\omega\left(k_{\mathrm{rec}}-\left(k_{\mathrm{ps}}\left(k_{\mathrm{ps}}+k_{\mathrm{rec}}\right)-\omega^{2}\right) R C\right)}{\left(k_{\mathrm{ps}}+k_{\mathrm{rec}}-\omega^{2} R C\right)+\left(\left(k_{\mathrm{ps}}+k_{\mathrm{rec}}+\frac{1}{R C}\right) \omega R C\right)^{2}}
$$

The fitting of the real and the imaginary part of the photocurrent was carried out in accordance with the model derived in ref. 34. The assumption of the model is that the electrochemical cell has a non-faradaic contribution due to a capacitor in series with a resistor (capacitance of the interface in combination with the total solution resistance resulting in an $R C$ circuit). That gives insight into four parameters controlling the photocurrent response at the interface, i.e. the interface $R C$ constant $(R C)$, the electron injection rate $(g)$, the product separation rate constant $\left(k_{\mathrm{ps}}\right)$ and the product recombination rate constant $\left(k_{\mathrm{rec}}\right)$. The $R C$ parameter reflects the relaxation through the resistor of the capacitor, consisting of two back-to-back double layers with the excess charges brought by the oxidized/ reduced electron donor/acceptor, i.e. the "successor complex" in the Marcus theory of electron transfer.

The potential dependence of the $R C$ time constant of the interface (data not shown in the present article) has a quadratic shape with a minimum near $\Delta_{\mathrm{o}}^{\mathrm{w}} \phi=$ 0 , as expected from the Gouy-Chapman model. ${ }^{\mathbf{4 0 , 4 1}}$ The resistance is independent of $\Delta_{\mathrm{o}}^{\mathrm{w}} \phi$, whereas an increase in $R C$ at positive and negative potential differences reflects the increase of the capacitance with the polarisation of the interface. The $R C$ time constant is a property of the interface and is not directly involved in the redox reactions; thus, the discussion will be limited to the other parameters.

Fig. 2 demonstrates the potential-dependence of the other fitted parameters, such as the steady-state current $(j$ photo $), g, k_{\mathrm{ps}}$ and $k_{\text {rec }}$. According to the general theory of photo-induced ET at the ITIES developed by Fermin et al. ${ }^{34}$ and Samec et al. ${ }^{35}$ the electron injection rate is defined as:

$$
g=e k_{\mathrm{et}} \Gamma_{\mathrm{s}}^{*}=e k_{\mathrm{et}} \frac{I \sigma}{k_{\mathrm{et}}+k_{\mathrm{d}}} \Gamma_{\mathrm{s}}
$$

where $e$ is the elementary charge, $\Gamma_{\mathrm{s}}^{*}$ is the surface density of excited porphyrins, $\Gamma_{\mathrm{s}}$ is the surface density of adsorbed porphyrins, $k_{\mathrm{d}}$ is the de-excitation rate constant of the excited porphyrin, $I$ is the photon flux at the interface and $\sigma$ is the absorption cross-section of the porphyrin.

The electron transfer rate constant $\left(k_{\mathrm{et}}\right)$ in eqn (3) exponentially depends on $\Delta_{\mathrm{o}}^{\mathrm{w}} \phi$ according to the following equation:

$$
k_{\text {et }}=k_{\text {et }}^{\prime} \exp \left(\frac{\alpha_{\text {et }} F \Delta_{\mathrm{o}}^{\mathrm{w}} \phi}{R T}\right)
$$

where $k_{\text {et }}^{\prime}$ is the rate constant at zero potential difference, $F$ is the Faraday constant, $R$ is the ideal gas constant, and $T$ is the temperature. The parameter $\alpha_{\text {et }}$ is the charge transfer coefficient of the Butler-Volmer equation, ${ }^{\mathbf{1 4}, \mathbf{1 7}}$ reflecting the fraction of the potential difference that is effectively felt by the donor-acceptor couple.

Therefore, in the present work, where both the donor and acceptor are neutral species, the parameter $g$ is potential-dependent only due to $k_{\mathrm{et}}$, whereas $\Gamma_{\mathrm{s}}^{*}$ and $\Gamma_{\mathrm{s}}$ are potential-independent. Thus, contrary to the results previously published in ref. 34 and 37, we were able to extract the electron transfer rate constant and the 

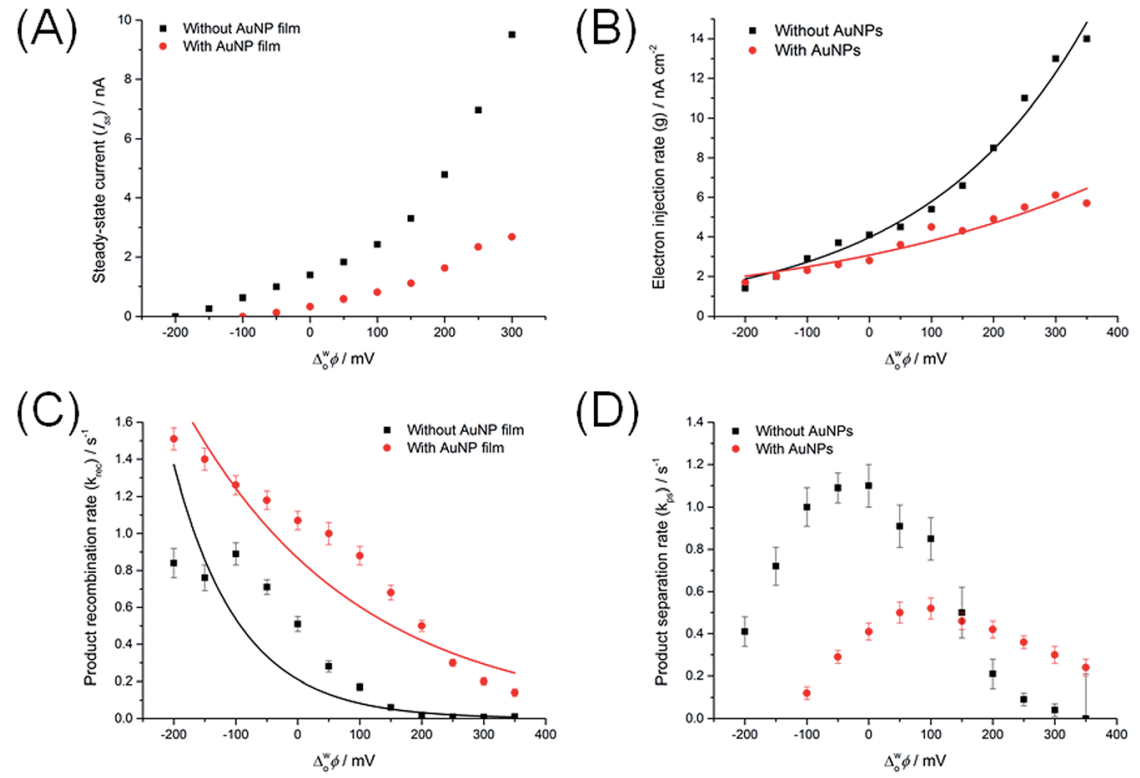

Fig. 2 IMPS results and their curve fit with the existing model of the photocurrent at the ITIES. (A) A steady-state current (jphoto), taken as j $j_{\text {photo, }}^{\text {is }}$ for the lowest frequency versus Galvani potential difference across the ITIES. (B) Electron injection parameter ( $g$ ) for the system described in Scheme 2, fitted with eqn (1) and (2). (C) Product recombination rate constant for the system described in Scheme 2, fitted with eqn (3). (D) Product separation rate constant for the system described in Scheme 2. For all panels the dots represent experimental data and solid lines show the curve fitting according to the corresponding equation.

charge transfer coefficient by fitting the curves in Fig. 2B with eqn (3) and (4), with $\sigma=10^{-17} \mathrm{~cm}^{2}, I=1.3 \times 10^{16} \mathrm{~cm}^{-2} \mathrm{~s}^{-1}$ and $k_{\mathrm{d}}=10^{5} \mathrm{~s}^{-1}$. In fact, the electron injection clearly shows an exponential dependence on the Galvani potential difference. These parameters are summarized in Table 1.

As expected and shown in our previous publications, ${ }^{\mathbf{1 4}, 17}$ the parameter $\alpha$ in the presence of the AuNP film was very close to zero, whereas $k_{\text {et }}$ increased twice in comparison to the bare ITIES. Conversely, the $\Gamma_{\mathrm{s}}$ decreased, reflecting that a part of the interface was blocked by the presence of AuNPs. However, this smaller surface density is counterbalanced by a higher $k_{\text {et }}$ value. The compensation of a lower density by faster kinetics is seen at low potential differences, where the $g$ values, with and without the film, are similar (Fig. 2B). In the case of the gold nanoparticle film, a smaller $\alpha$ lowers the increase of the potential difference and, thus, leads to the divergence of the $g$ values of the two systems.

Table 1 Summary of the system parameters $\left(\alpha_{\mathrm{et}}, \Gamma_{\mathrm{s}}\right.$ and $\left.k_{\mathrm{et}}\right)$ obtained from fitting the experimental data with eqn (3) and (4)

\begin{tabular}{lll}
\hline & Bare ITIES & ITIES with AuNP film \\
\hline$\alpha_{\mathrm{et}}$ & 0.13 & 0.08 \\
$k_{\mathrm{et}} / \mathrm{s}^{-1}$ & $4.3 \times 10^{4}$ & $9.4 \times 10^{4}$ \\
$\Gamma_{\mathrm{s}} / \mathrm{m}^{-2}$ & $1.5 \times 10^{12}$ & $6.2 \times 10^{11}$
\end{tabular}


Further, we will consider the potential-dependence of $k_{\mathrm{rec}}$ and $k_{\mathrm{ps}}$. The potential-dependence of the product recombination rate constant $\left(k_{\mathrm{rec}}\right)$, with and without the nanofilm, is presented in Fig. 2C. An equation similar to eqn (1) can be written to fit the obtained data in agreement with the previously mentioned general theory of the photocurrent at the ITIES:

$$
k_{\mathrm{rec}}=k_{\mathrm{rec}}^{\prime} \exp \left(-\frac{\alpha_{\mathrm{rec}} F \Delta_{\mathrm{o}}^{\mathrm{w}} \phi}{R T}\right)
$$

In this case, the minus sign reflects the intrinsic nature of the photoelectrochemical process: with an increasing Galvani potential difference the product recombination rate constant should effectively decrease because the activation energy of the reverse process has been increased. The activation energies of the electron transfer and electron recombination are in principle interdependent, and in theory, the amount by which the electron transfer activation energy is lowered is the same as that by which the electron recombination activation energy is increased. This corroborates the previously obtained results. ${ }^{37}$

The obtained $\alpha_{\text {rec }}$ and $k_{\text {rec }}$ values are represented in Table 2 . Their values show similar trends to those observed in Table 1: very low $\alpha$ values and a higher rate constant in the presence of the nanofilm.

Both the increased electron transfer and recombination rate constants are contradictory in the context of the Marcus theory of electron transfer. ${ }^{42}$ Indeed, in this theory, when a redox reaction is favoured in one direction by a shift in the Gibbs free energy - and consequently a shift of the activation energy - it should slow down for the reverse reaction. Thus, the observed changes in the electron transfer and recombination rate constants with the nanoparticles imply that the kinetics are modified because the reaction mechanism is changed and not only because the effective potential difference felt by the redox species is different. Thus, one can conclude that the reaction is catalyzed by the nanoparticles.

Most likely the first step is a rapid equilibration of the NP Fermi level with that of the organic phase redox couple (DMFc). As DMFc is present in excess, the Fermi level of this phase is fixed by the redox potential of the DMFc ${ }^{+} / \mathrm{DMFc}$ couple. ${ }^{\mathbf{4 3 , 4 4}}$ Thus, the gold nanoparticles become a "reservoir of electrons" for further photoelectrochemical reactions but with a redox potential that is tuned by the Fermi level of the redox couple in the organic phase. As the Fermi level difference between the aqueous and organic phases depends on the Galvani potential difference, the Fermi level of the AuNPs can be controlled by changing the Galvani potential difference across the interface (Scheme 1). ${ }^{\mathbf{1 4 , 1 7}}$

Thus, the electron transfer, $k_{\text {et }}$, and recombination, $k_{\text {rec }}$, rate constants measured using IMPS are not related to the transfer of an electron between the donor and the acceptor but rather to the transfer between the donor or the

Table 2 Summary of the system parameters $\left(\alpha_{\text {rec }}\right.$ and $\left.k_{\text {rec }}\right)$ obtained from fitting the experimental data with eqn (5)

\begin{tabular}{lll}
\hline & Bare ITIES & ITIES with AuNP film \\
\hline$\alpha_{\text {rec }}$ & 0.12 & 0.07 \\
$k_{\text {rec }} / \mathrm{s}^{-1}$ & 0.40 & 0.94
\end{tabular}


acceptor and the "electron reservoir", namely, the gold nanoparticles. As the equilibration of the Fermi level of the nanoparticles and that of the organic phase is assumed to be fast, the reaction that is measured is probably the one taking place between the porphyrin and the nanoparticles.

From a thermodynamic point of view, the interaction between the porphyrin and the nanoparticles is equivalent to the one taking place directly between ZnDMPyDSPP and DMFc because their driving forces are similar, as the Fermi level of the nanoparticles is fixed by the redox potential of DMFc. Nevertheless, as the electron transfer from a gold nanoparticle to a porphyrin molecule takes place from a continuum of states for the metal to the discrete levels of a molecule, one can expect faster kinetics for the transfer and recombination processes. However, in the absence of direct measurements of the electron transfer kinetics from the donor/acceptor and the nanoparticles, it is difficult to say with certainty whether the limiting reaction is the transfer from the donor to the nanoparticles or the transfer from the nanoparticles to the acceptor.

Finally, the product separation rate constant $\left(k_{\mathrm{ps}}\right)$ can be extracted from the fitting of the Nyquist plots of the photocurrent response (Fig. 2D). For both systems the curves present a maximum near $\Delta_{\mathrm{o}}^{\mathrm{w}} \phi=0$, and decrease with the positive and negative polarisation, suggesting a dependence on double layer effects. Nevertheless, with the nanoparticles, the curve maximum is shifted by roughly $100 \mathrm{mV}$ towards more positive potential differences compared to that for the bare interface.

In the considered model and for truly heterogeneous electron transfer, $k_{\mathrm{ps}}$ is related to the separation of the successor complex. In the presence of the nanoparticles, it describes, most likely, desorption of the reduced (negatively charged) porphyrin after the electron transfer reaction. In these two cases, the double layers are different: two back-to-back Gouy-Chapman double layers for the case of the reaction at the ITIES and a single Gouy-Chapman double layer at the

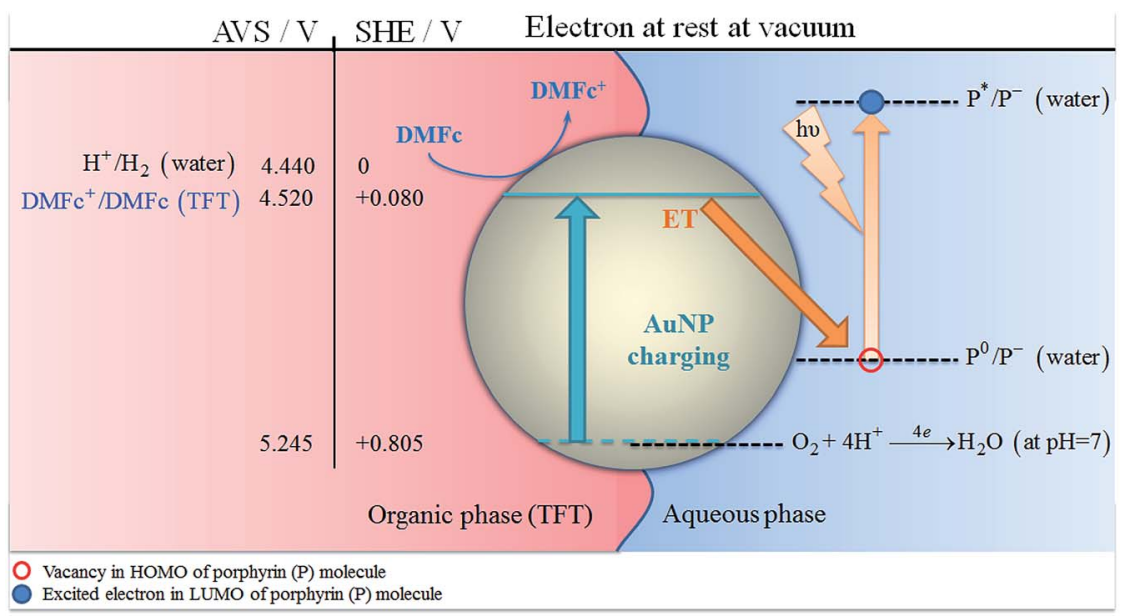

Scheme 1 Equilibration of the Fermi levels between the gold nanoparticles and redox couples in both phases. Immediately after contact of the nanoparticles with the electron donor (DMFC) the Fermi level of the gold nanoparticle equilibrates with its redox potential (AuNP charging). Excitation of the porphyrin molecule leads to the formation of a vacancy in the HOMO, which is refilled with an electron from a gold nanoparticle (electron transfer, ET). 
nanoparticle surface in the case of the nanofilm. Thus, the observed shift of the $k_{\mathrm{ps}}$ peak may reflect the changes in the double layers.

Also, this observation is consistent with the trends of the electron transfer and recombination rate constants suggesting that the measured reaction was taking place between the nanoparticles and the porphyrin.

Effect of plasmon enhancement on the observed photocurrent. As we mentioned in the beginning, one of the goals of these experiments was to revisit the plasmonic enhancement of the photocurrent, previously obtained by Schaming et al. ${ }^{37}$ In the above subsection we clearly demonstrated with the IMPS technique that the nanoparticles adsorbed at the ITIES mainly changed the kinetics of the interfacial reactions in an interfacial redox electrocatalytic process. ${ }^{17,18,43,44}$

Most likely, the film preparation method in ref. 37 , i.e. the addition of large amounts of methanol and emulsification of the phases containing both redox phases, led to the formation of a thick (micrometer scale) and dense layer at the interface, where all the components are mixed together. Therefore, the real plasmonic effects could probably be hidden by these modifications.

As emphasized in the previous paragraph, the possibility of an enhancement of the photocurrent at the liquid-liquid interface through plasmonic resonance is still an open question. Nevertheless, the present work shows that increasing the electron transfer efficiency is possible at least through direct interfacial redox electrocatalysis. The overall smaller current densities with the nanoparticles are explained only by a lower surface coverage of the photoactive species and a smaller charge transfer coefficient. Thus, at equal surface densities, the presence of gold nanoparticles does not enhance the photocurrent compared to the bare interface. Thus, if the limitations brought by the decrease in surface density can be overcome, the use of gold nanoparticles could be a valuable way to increase the efficiency of the electron transfer at polarized liquid-liquid interfaces.

\section{Marangoni-type shutter based on gold nanoparticle assemblies at the ITIES}

The aim of this part of the paper is to show how varying the Galvani potential difference may give control over the position of the nanoparticles at the ITIES.

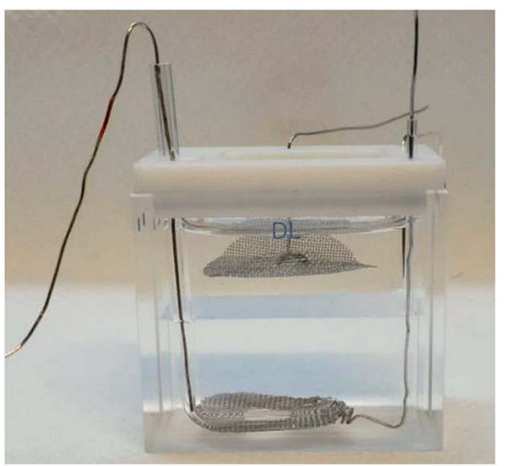

Fig. 3 Silanized four-electrode electrochemical cell with an extremely flat and smooth interface. 
We developed a method to silanize a quartz cell in order to achieve an ultimately flat interface, as presented in Fig. 3. In this case, the use of a flat interface is important to eliminate the effects of the gravitational force, and focus only on the changes brought about by the electric field.

Blank experiments. All necessary blank experiments were carefully carried out and checked to confirm the obtained results. For the reflectance experiments, a green laser with a wavelength of $532 \mathrm{~nm}$ was selected. The recorded angular dependence of the reflectance from a bare water-TFT agrees very well with the theoretically predicted one. The estimated value of the critical angle for the total internal reflectance (TIR) conditions is $\theta_{\mathrm{TIR}}^{\exp }=70^{\circ}$ (Fig. 4, the black curve), whereas the theoretical value is $\theta_{\mathrm{TIR}}^{\text {theor }}=70.05^{\circ}$.

Reflectance experiments with sodium dodecyl sulfate (SDS): on the way to Marangoni-shutters. The addition of SDS decreased the overall intensity of the reflected light above the critical angle by $c a .10 \%$, whereas the subsequent addition of the gold nanoparticles into the cell did not affect the reflectance (Fig. 4, red and blue curves). Notably, the normalization of the reflectance was carried out with respect to the maximal intensity at the bare water-TFT interface. There are two possible contributions to the drop in the intensity above the critical angle by roughly 10\%: (i) scattering and/or absorbance of the light by SDS molecules and (ii) instability of the diode laser intensity over time. Based on the observed angular dependence, two angles below $\left(66^{\circ}\right)$ and above $\left(72.4^{\circ}\right) \theta_{\text {TIR }}$ were chosen to investigate the influence of the electric field on the reflectance of gold nanoparticle assemblies.

The data recorded at these two angles are presented in Fig. 5A and B, while alternating the electric field across the interface using cyclic voltammetry. The scan rate in both cases was $25 \mathrm{mV} \mathrm{s}^{-1}$. Changes in the reflected light intensity occurred at both edges of the potential window, however, the intensity increased much more at the negative end of the potential window, where anions $\left(\mathrm{A}^{-}\right)$ transfer across the ITIES from water into the organic phase. A similar behaviour was also observed above the critical angle (Fig. 5B) during at least 9 cycles. Unfortunately, upon cycling, the system underwent an aggregation process, turning the solution color from red to bluish (Fig. 5C). Also, we found that gold nanoparticles were moving during the sweeping of the potential from the middle

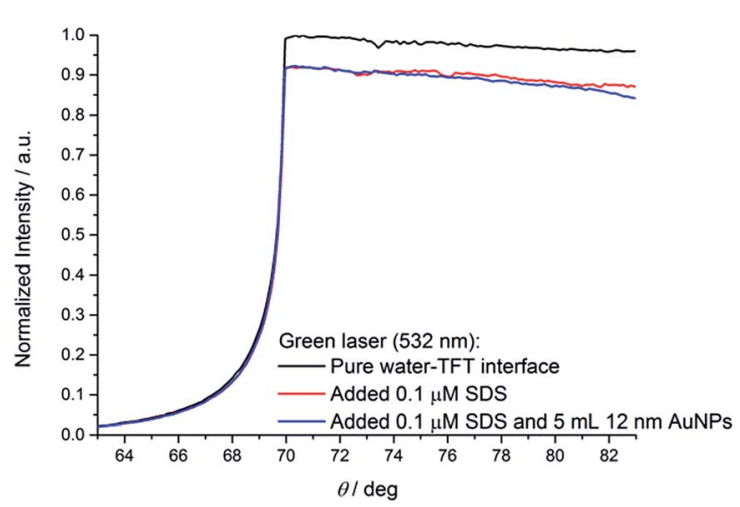

Fig. 4 Effect of sodium dodecyl sulfate (SDS) and $12 \mathrm{~nm}$ gold nanoparticles (AuNPs) on the angular dependence of the reflectance. All graphs were normalized to the intensity of the bare water-TFT interface (the black curve). 
of the cell to the corners and backward. Recently, Scanlon et al. have reported the observation of a similar effect in the case of floating carbon nanotubes at LLI. ${ }^{28}$

Notably, the reflectance oscillations upon transfer of the supporting electrolyte were recorded for the bare interface and the cell with only gold nanoparticles without SDS. However, the amplitude of the changes was extremely small, below $2 \%$ of the overall intensity. Additionally, similar behavior was observed in the case of the SDS covered interface without gold nanoparticles.

Thus, the addition of ionic surfactant molecules is a critical step for the Marangoni-type shutters.

Finally, we show the Marangoni-type shutter in action. Fig. 6A demonstrates snapshots from a video recorded during the testing of the Marangoni-type shutter at $66^{\circ}$ (below the critical angle $\theta_{\text {TIR }}$ ). This set of snapshots clearly illustrates how the reflected laser intensity increased and decreased because the gold nanoparticles move in and out the spot of the laser at the ITIES.

In the middle of the polarizable potential window the gold nanoparticles were distributed around the perimeter of the cell. However, a portion of light reflected from the bare ITIES (Fig. 6A, bottom-left snapshot) remained at $66^{\circ}$, as shown in Fig. 4. Immediately after, anions began to transfer from the aqueous to the organic phase, and gold nanoparticles started to migrate to the middle region of the cell. They rapidly accumulated, causing a vast increase in the reflected light (Fig. 6A, top-right snapshot). The effect lasted as long as the transferred anions stayed in the organic phase ( $c a .20 \mathrm{~s})$. Then the system returned to its initial state with the gold nanoparticles spread around the perimeter of the cell (Fig. 6A, bottom-right snapshot).

(A)

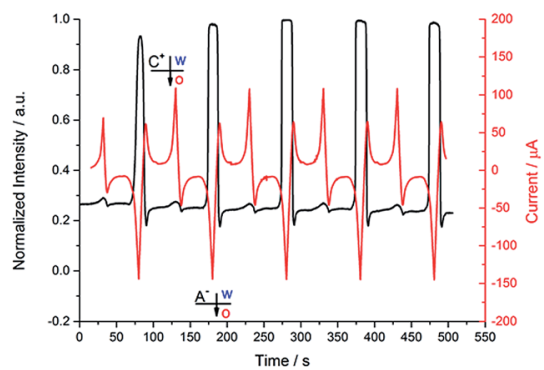

(B) $\quad \theta=72.4^{\circ}$

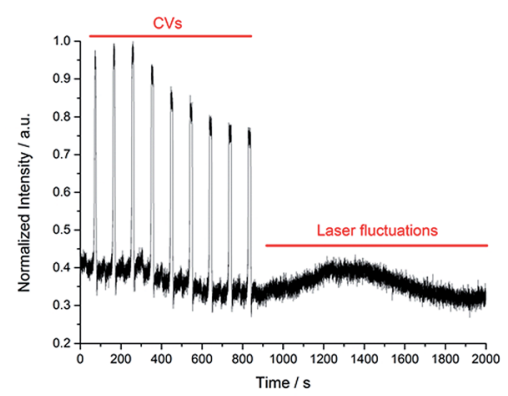

(C)

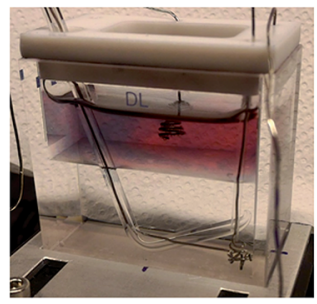

Fig. 5 Marangoni-type shutter at the ITIES driven using an electric field. (A) Change in the reflectance upon sweeping the potential at $\theta=66^{\circ}$ (below the critical angle $\theta_{T I R}$ ). (B) Similar effect observed for $\theta=72.4^{\circ}$ (above the critical angle $\theta_{\mathrm{TIR}}$ ). (C) An electrochemical cell after several tens of cycles, the blueish color of the gold nanoparticle solution indicates degradation of the system. 
(A)

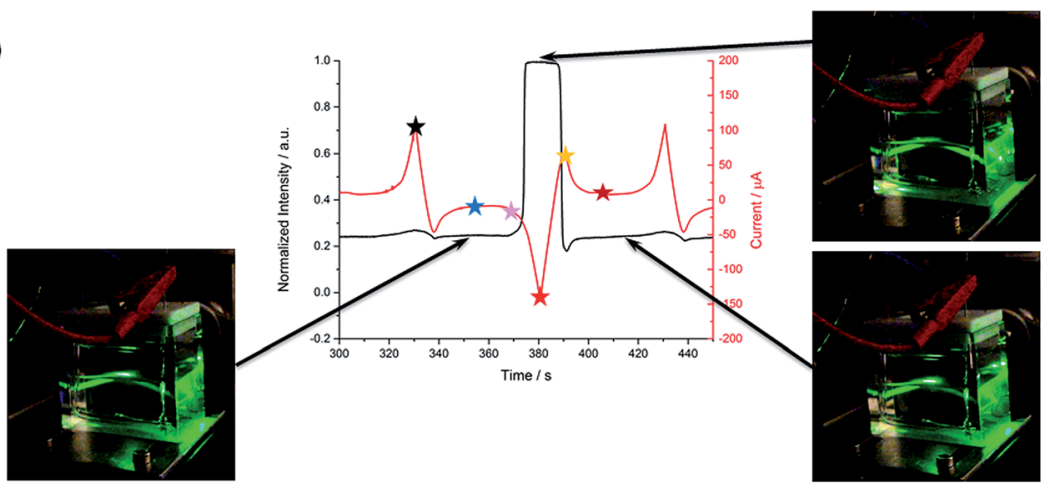

(B)

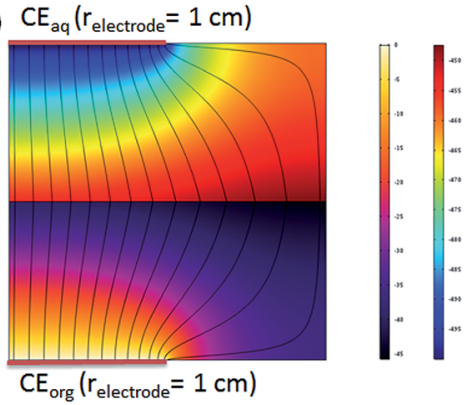

(C)

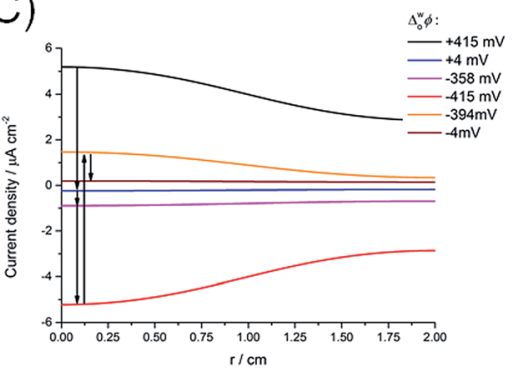

Fig. 6 Marangoni-type shutter in action. (A) A part of the cyclic voltammogram with the corresponding snapshots taken from the recorded video. The stars in panel (A) correspond to the curves in panel (C). Results of the COMSOL simulation for (B) the electric field distribution at $\Delta_{\circ}^{\mathrm{w}} \phi=-415 \mathrm{mV}$ and $(\mathrm{C})$ the current density distributions at various Galvani potential differences. $r$ denotes the distance from the center of the electrode. For clarity the position of the counter electrodes in both phases are shown.

Possible working principles of Marangoni-type shutters. There are three possible effects contributing to the behavior of the Marangoni-type shutters: (i) changes in the interfacial surface tension $(\gamma)$ upon applying a Galvani potential difference, (ii) instabilities caused by the transfer of surfactant molecules (SDS), and (iii) a non-uniform electric field distribution between the working electrodes and the ITIES.

In the case of the ITIES, the interfacial surface tension is a function of the applied Galvani potential difference due to an electrocapillary effect. ${ }^{45}$ These changes are expressed in a differential form as the Gibbs-Lippman equation: ${ }^{40}$

$$
\left(\frac{\partial \gamma}{\partial \Delta_{\mathrm{o}}^{\mathrm{w}} \phi}\right)_{\mu_{i}, T, i \neq j}=-q^{\mathrm{w}}+\sum_{j=1}^{n} F Z_{j} \Gamma_{j}^{\mathrm{o}}-\sum_{j=1}^{n}\left(\Gamma_{j}^{\mathrm{o}}+\Gamma_{j}^{\mathrm{w}}\right)\left(\frac{\partial \mu_{j}^{\mathrm{w}}}{\partial \Delta_{\mathrm{o}}^{\mathrm{w}} \phi}\right)_{T}
$$

where $\gamma$ is the interfacial surface tension, $\Delta_{\mathrm{o}}^{\mathrm{w}} \phi$ is the Galvani potential difference, $q^{\mathrm{w}}$ is the surface charge density in the diffuse layer of the aqueous phase, $Z_{j}$ is the charge of ion $j, \Gamma_{j}^{\mathrm{o}, \mathrm{w}}$ is the surface excess of ion $j, F$ is the Faraday constant, $\mu_{j}^{\mathrm{w}}$ is the electrochemical potential of ion $j$, whereas $\mathrm{w}$ and o denote the aqueous and organic phases, respectively.

For 1 : 1-electrolytes, eqn (6) can be transformed into eqn (7): ${ }^{11}$ 


$$
\gamma=A \cosh \left(\frac{F\left(\Delta_{\mathrm{o}}^{\mathrm{w}} \phi-\Delta_{\mathrm{o}}^{\mathrm{w}} \phi_{\mathrm{PZC}}\right)}{2 R T}\right)+B
$$

where $\Delta_{\mathrm{o}}^{\mathrm{w}} \phi_{\text {PZC }}$ is the applied potential at the point of zero charge (PZC) or the electrocapillary maximum. $A$ and $B$ are integration constants.

The electrocapillary effect described by eqn (6) and (7) lowers the interfacial surface tension, when the ITIES is polarized, and establishes a maximum when $\Delta_{\mathrm{o}}^{\mathrm{w}} \phi=\Delta_{\mathrm{o}}^{\mathrm{w}} \phi_{\mathrm{PzC}}$. Thus, if the observed displacement of the nanoparticles is related only to the variations in the interfacial surface tension with the applied potential, then it should be continuous over all the available polarizations of the ITIES. However, the major changes in the reflected light intensity were recorded only on one side of the polarizable potential window, where the transfer of the anions (including SDS) occurred. The changes are sharp and coincide quite well with the transfer of the anions.

Therefore, we should also consider the transfer of SDS molecules across the ITIES. Changing the surface coverage with an electric field could cause the Marangoni instabilities that lead to the spontaneous oscillation of the interfacial surface tension. ${ }^{46-48}$ Recently, Kovalchuk has reviewed this topic with the absence of the electric field. ${ }^{49}$

And last but not least, a non-uniform electric field distribution could significantly influence the nanoparticle assemblies when the interfacial surface tension is significantly reduced. The platinum mesh electrodes did not cover the entire surface area of the interface, causing the non-uniform distribution of the electric field at the ITIES, as shown with the COMSOL simulation (see the electric field profiles in Fig. 6B and C). The results show identical current distribution for the negative and positive ends of the potential window. Hence, the non-uniform electric field distribution cannot be solely responsible for the movement of the particles observed only at the negative end of the potential window.

Nevertheless, we demonstrated for the first time a working prototype of a Marangoni-type shutter. However, the true working mechanism of the presented Marangoni-type shutter still remains unclear and more follow-up work is needed to reveal it. Also, further development of this type of electrically driven interfacial plasmonic device should be carried out in terms of the overall stability and reliability of the system.

\section{Conclusions}

In this article we have shown that the polarizable ITIES is a promising platform to control and manipulate gold nanoparticle assemblies, leading to "smart" electrovariable nanosystems.

In one series of experiments, the intrinsic properties of the nanoparticles at the ITIES were tuned using an external electric field. Thus, the adsorption of gold nanoparticles at the ITIES was a valuable way to control the interfacial electron transfer reaction through "interfacial redox electrocatalysis". The Fermi level of the nanoparticles was fixed by the organic phase redox couple, thus leaving the thermodynamics of the overall reaction unchanged, while the kinetics were doubled because the reaction took place between the nanoparticles and the aqueous phase photoredox species. Using a more reproducible cell preparation 
method, as well as modern IMPS apparatus, we have shown that plasmonic effects giving rise to an enhancement of the photocurrent were unlikely, or at most of limited impact compared to the catalytic effect of the nanoparticles. Nevertheless, the possibility of such an effect could not be completely ruled out. Overall, the use of metal nanoparticles appears to be an interesting tool to improve the efficiency of redox reactions taking place at the liquid-liquid interface.

In another series of experiments, we observed that nanoparticles at the ITIES could be positioned with an external electric field. We have shown an encouraging example of a working Marangoni-type shutter at the ITIES, which is based on the displacement of the gold nanoparticles in the plane of the ITIES. The presented shutter demonstrated moderate stability upon cycling, and could be switched on and off for at least tens of cycles.

\section{Experimental}

\section{Chemicals}

All chemicals were used as received without further purification. Lithium chloride 99.0\% and tetramethyl ammonium chloride $>99.0 \%$ were purchased from Fluka, trisodium citrate dihydrate $\left(\mathrm{Na}_{3} \mathrm{C}_{6} \mathrm{H}_{5} \mathrm{O}_{7} \cdot 2 \mathrm{H}_{2} \mathrm{O}, 98 \%\right)$ and $\alpha, \alpha, \alpha$-trifluorotoluene 99+\% (TFT) were received from Acros, hydrogen tetrachloroaurate(III)trihydrate $\left(\mathrm{HAuCl}_{4} \cdot 3 \mathrm{H}_{2} \mathrm{O}, 99.999 \%, 49 \% \mathrm{Au}\right)$ was purchased Alfa Aesar, ascorbic acid $\left(\mathrm{C}_{6} \mathrm{H}_{8} \mathrm{O}_{6}\right)$ was obtained from Riedel-de-Haem, bis(pentamethylcyclopentadienyl) iron(II) (DMFc) was received from STREM and sodium dodecyl sulfate (SDS) was purchased from Sigma.

The organic phase supporting electrolyte, bis(triphenylphosphoranylidene) ammonium tetrakis(pentafluorophenyl)borate (BATB), was prepared through metathesis of aqueous equimolar solutions of BACl and LiTB-DEE purum. The resulting precipitates were filtered, washed, and recrystallized from an acetone : methanol $(1: 1)$ mixture. $^{34}$

The porphyrin used in this study, zinc(II) 5,15-(di-4-sulfonatophenyl)-10,20-(di$N$-methyl-4-pyridyl)porphyrin disodium dichloride (ZnDMPyDSPP), was obtained from PorphyChem (France) using a custom synthesis. As the compound contained non water soluble impurities, the product was first dissolved in water and filtered through $0.22 \mu \mathrm{m}$ HPLC PTFE filters (Infochroma AG) before use. The final concentration of the porphyrin was measured using UV-vis absorption spectroscopy.

\section{Synthesis of AuNPs}

A solution of colloidal gold nanoparticles was prepared through the reduction of $\mathrm{HAuCl}_{4} \cdot 3 \mathrm{H}_{2} \mathrm{O}$ in water with trisodium citrate $\left(\mathrm{Na}_{3} \mathrm{Citr}\right)$, as reported by Turkevich and further developed by Frens. ${ }^{50,51}$ Briefly, $41.5 \mathrm{mg}$ of $\mathrm{HAuCl}_{4} \cdot 3 \mathrm{H}_{2} \mathrm{O}$ was dissolved in $300 \mathrm{~mL}$ of deionized water and heated to $100{ }^{\circ} \mathrm{C}$ in a round-bottomed flask with stirring. $9 \mathrm{~mL}$ of a $1 \% \mathrm{w} / \mathrm{v} \mathrm{Na}_{3}$ Citr solution was injected to prepare the smaller 12-14 $\mathrm{nm}$ gold nanoparticles. After approximately 30 seconds the solution initially turned dark black, before changing to red. The solution was maintained at its boiling point for 45 minutes and subsequently cooled down to generate a stable colloidal suspension. 


\section{Four-electrode electrochemical setup}

All electrochemical measurements were performed in a four-electrode electrochemical cell with an Autolab PGSTAT 204 potentiostat (Metrohm, Switzerland).

Cyclic voltammetry at a soft interface. The typical composition of the electrochemical cell is described in Scheme 2. When necessary, a gold nanoparticle film was deposited at the liquid-liquid interface following a procedure recently published by our group. ${ }^{14,18}$

All electrochemical measurements were carried out under a nitrogen atmosphere in order to prevent any interactions of the system with oxygen, i.e. the quenching of the excited state of the porphyrin or direct oxygen reduction with DMFc. ${ }^{18}$

IMPS measurements. To measure IMPS the Autolab PGSTAT 204 potentiostat was equipped with an Autolab LED driver (Metrohm, Switzerland). The LED selected for this study had an emission spectrum with a central wavelength of 470 $\mathrm{nm}$ and a full band width of $26 \mathrm{~nm}$. The interface was illuminated from the bottom of the cell, i.e. through the organic phase, and at a distance of $c a .5 \mathrm{~cm}$ from the LED. The light intensity was varied by $10 \%$. The cell composition is also given in Scheme 2.

\section{Marangoni shutters}

Scheme 3 shows the electrochemical cell composition used to study the Marangoni-type shutter at a soft interface. As LiCl salt at a concentration of $10 \mathrm{mM}$

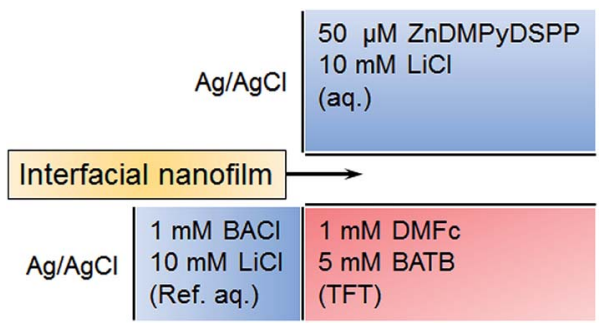

Scheme 2 Typical electrochemical cell composition with and without a gold nanoparticle film.

\begin{tabular}{r|l|l}
\multicolumn{1}{l||}{} & $\begin{array}{l}\text { Some residual ions } \\
\text { Ag/AgCl }-0.1 \mu \mathrm{M} \\
12 \mathrm{~nm} \text { AuNPs (+citr) } \\
\text { (aq.) }\end{array}$ \\
\hline Ag/AgCl & $\begin{array}{l}1 \mathrm{mM} \mathrm{BACl} \\
10 \mathrm{mM} \mathrm{LiCl} \\
\text { (aq.) }\end{array}$ & $\begin{array}{l}10 \mathrm{mM} \text { BATB } \\
\text { (TFT) }\end{array}$ \\
\hline
\end{tabular}

Scheme 3 Composition of the electrochemical cell used to show the Marangoni-type shuttering effect at the ITIES. AuNPs denotes gold nanoparticles, whereas citr denotes the remaining citrate molecules from the synthesis. 
significantly affects the stability of the gold nanoparticles, only residual ions and SDS molecules were used to establish electrical conductance in the aqueous phase. A concentrated solution of SDS was added to the aqueous phase, mixed and left for half an hour to let the SDS molecules settle and organize at the interface. Then, half of the water was substituted with a solution of $12 \mathrm{~nm}$ AuNPs.

Concentration of SDS was chosen as $0.1 \mu \mathrm{M}$ to ensure rapid sorption and complete coverage of the ITIES with surfactant molecules.

\section{Angle-dependent reflectance measurements}

The reflectance of the ITIES at different incidental angles was measured using a home-made "arms-setup", which is presented in Fig. 7. A green (532 nm) diodelaser was used in all the reflectance experiments.

\section{Simulations for the current distribution}

The secondary current distribution in the electrochemical cell used to study the Marangoni-type shutter was simulated with COMSOL Multiphysics 5.2a. The geometry of the cell was approximated as a $2 \mathrm{~cm}$ radius cylinder in 2D-axis symmetry, where electrodes of $1 \mathrm{~cm}$ in radius were placed $1 \mathrm{~cm}$ above and below the ITIES, as shown in Fig. 6B. The potential distribution was solved from the Ohm's law for both phases, with the following equation:

$$
J=\sigma_{\mathrm{i}} E=-\sigma_{\mathrm{i}} \nabla \phi_{\mathrm{i}}
$$

where $J$ is the current density flux, $E$ is the electric field and $\sigma_{\mathrm{i}}$ and $\phi_{\mathrm{i}}$ are the conductivity and Galvani potential of the phase i. $\sigma_{\mathrm{w}}$ was measured as $145 \mu \mathrm{S} \mathrm{cm}^{-1}$ and $\sigma_{\mathrm{o}}=167 \mu \mathrm{S} \mathrm{cm}^{-1}$. The forward and backward transfer (water to oil, oil to water) of ions was described with Butler-Volmer type expressions, where the rate constants were expressed as: ${ }^{17}$

$$
k_{\mathrm{i}, \mathrm{f}}=k_{\mathrm{i}}^{0} \mathrm{e}^{\frac{\alpha z_{\mathrm{i}} F}{R T}\left(\phi_{\mathrm{w}}-\phi_{\mathrm{o}}-\Delta \phi_{\mathrm{i}}^{0}\right)}
$$

(A)

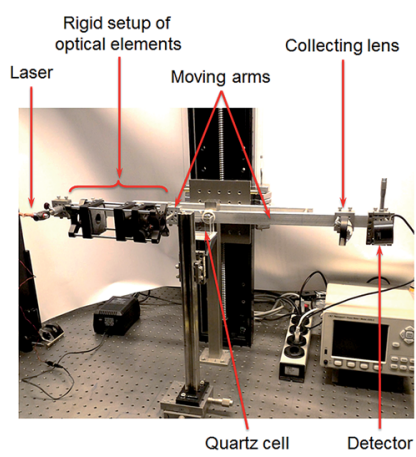

(B)

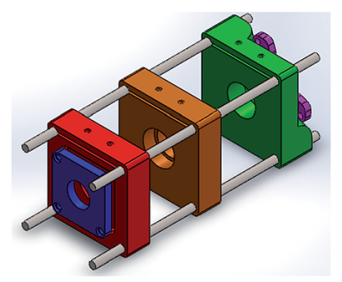

Fig. 7 Design of a home-made arms setup to investigate the angular dependence of the laser reflectance from interfacial nanofilms. (A) The setup consisted of simultaneously moving "arms", a laser light source, the necessary optical components and a detector. (B) A close view of the 3D-printed parts of the optical component holder: the $\lambda / 2$ plate (redblue), the polarizer (orange) and the aperture (green-pink) to control the spot size. 


$$
k_{\mathrm{i}, \mathrm{b}}=k_{\mathrm{i}}^{0} \mathrm{e}^{\frac{(\alpha-1) z_{\mathrm{i}} F}{R T}\left(\phi_{\mathrm{w}}-\phi_{\mathrm{o}}-\Delta \phi_{\mathrm{i}}^{0}\right)}
$$

The concentrations of ions were calculated using Fick's second law. This approach was used instead of the more rigorous Nernst-Planck equation, as the solution of the tertiary current distribution is computationally more intensive. The simulation took $c a$. two days with Intel Core quad-core i-7-4870HQ CPU @ $2.50 \mathrm{GHz}$, with $16 \mathrm{~GB}$ of RAM (MacBook Pro running Windows 7 as the operating system). However, less accurate simulations could be performed in 1 to $3 \mathrm{~h}$, depending on the density of the mesh, leading to sometimes significant inaccuracies and oscillations in the boundary fluxes.

\section{Acknowledgements}

Authors acknowledge Dr Dennison Christopher Raymond for fruitful discussion of the manuscript and the Swiss National Science Foundation (Grant: Solar Fuels 2000-20_152 557/1) for the financial support.

\section{References}

1 F. Bresme and M. Oettel, J. Phys.: Condens. Matter, 2007, 19, 413101.

2 B. P. Binks, Curr. Opin. Colloid Interface Sci., 2002, 7, 21-41.

3 J. B. Edel, A. A. Kornyshev, A. R. Kucernak and M. Urbakh, Chem. Soc. Rev., 2016, 45, 1581-1596.

4 W. H. Binder, Angew. Chem., Int. Ed., 2005, 44, 5172-5175.

5 J. B. Edel, A. a. Kornyshev and M. Urbakh, ACS Nano, 2013, 7, 9526-9532.

6 E. Smirnov, M. D. Scanlon, D. Momotenko, H. Vrubel, M. A. Méndez, P.-F. Brevet and H. H. Girault, ACS Nano, 2014, 8, 9471-9481.

7 E. Smirnov, P. Peljo, M. D. Scanlon, F. Gumy and H. H. Girault, Nanoscale, 2016, 8, 7723-7737.

8 M. E. Flatte, A. A. Kornyshev and M. Urbakh, Proc. Natl. Acad. Sci. U. S. A., 2008, 105, 18212-18214.

9 M. E. Flatté, A. A. Kornyshev and M. Urbakh, J. Phys.: Condens. Matter, 2008, 20, 73102.

10 M. E. Flatte, A. A. Kornyshev and M. Urbakh, J. Phys. Chem. C, 2010, 114, 17351747.

11 J.-P. Abid, M. Abid, C. Bauer, H. H. Girault and P.-F. Brevet, J. Phys. Chem. C, 2007, 111, 8849-8855.

12 B. Su, J.-P. Abid, D. J. Fermin, H. H. Girault, H. Hoffmannová, P. Krtil and Z. Samec, J. Am. Chem. Soc., 2004, 126, 915-919.

13 M. D. Scanlon, P. Peljo, M. A. Méndez, E. Smirnov and H. H. Girault, Chem. Sci., 2015, 6, 2705-2720.

14 E. Smirnov, P. Peljo, M. D. Scanlon and H. H. Girault, ACS Nano, 2015, 9, 65656575.

15 P. S. Toth, A. N. J. Rodgers, A. K. Rabiu, D. Ibañez, J. X. Yang, A. Colina and R. A. W. Dryfe, J. Mater. Chem. A, 2016, 4, 7365-7371.

16 P. S. Toth, A. N. J. Rodgers, A. K. Rabiu and R. A. W. Dryfe, Electrochem. Commun., 2015, 50, 6-10. 
17 P. Peljo, E. Smirnov and H. H. Girault, J. Electroanal. Chem., 2016, 779, 187198.

18 E. Smirnov, P. Peljo, M. D. Scanlon and H. H. Girault, Electrochim. Acta, 2016, 197, 362-373.

19 Z. Samec, V. Mareček, J. Weber and D. Homolka, J. Electroanal. Chem. Interfacial Electrochem., 1981, 126, 105-119.

20 Z. Samec, V. Mareček and J. Weber, J. Electroanal. Chem. Interfacial Electrochem., 1979, 96, 245-247.

21 S. Kihara, M. Suzuki, K. Maeda, K. Ogura, M. Matsui and Z. Yoshida, J. Electroanal. Chem. Interfacial Electrochem., 1989, 271, 107-125.

22 H. Hotta, S. Ichikawa, T. Sugihara and T. Osakai, J. Phys. Chem. B, 2003, 107, 9717-9725.

23 H. Tatsumi and H. Katano, Anal. Sci., 2007, 23, 589-591.

24 P. S. Toth, M. Velický, Q. M. Ramasse, D. M. Kepaptsoglou and R. A. W. Dryfe, Adv. Funct. Mater., 2015, 25, 2899-2909.

25 J. J. Nieminen, I. Hatay, P.-Y. P. Ge, M. A. Méndez, L. Murtomäki and H. H. Girault, Chem. Commun., 2011, 47, 5548-5550.

26 S. Rastgar, H. Deng, F. Cortés-Salazar, M. D. Scanlon, M. Pribil, V. Amstutz, A. A. Karyakin, S. Shahrokhian and H. H. Girault, ChemElectroChem, 2014, 1, 59-63.

27 I. Hatay, P. Y. Ge, H. Vrubel, X. Hu and H. H. Girault, Energy Environ. Sci., 2011, 4, 4246.

28 X. Bian, M. D. Scanlon, S. Wang, L. Liao, Y. Tang, B. Liu and H. H. Girault, Chem. Sci., 2013, 4, 3432.

29 H. L. T. Ho and R. A. W. Dryfe, Chem. Commun., 2011, 47, 11318-11320.

30 S. G. Booth, A. Uehara, S. Y. Chang, J. F. W. Mosselmans, S. L. M. Schroeder and R. A. W. Dryfe, J. Phys. Chem. C, 2015, 119, 16785-16792.

31 J. Zhang and P. R. Unwin, Phys. Chem. Chem. Phys., 2002, 4, 3820-3827.

32 H. Hotta, N. Akagi, T. Sugihara, S. Ichikawa and T. Osakai, Electrochem. Commun., 2002, 4, 472-477.

33 R. A. W. Dryfe, Z. Ding, R. G. Wellington, P. F. Brevet, A. M. Kuznetzov and H. H. Girault, J. Phys. Chem. A, 1997, 101, 2519-2524.

34 D. J. Fermin, H. Dung Duong, Z. Ding, P.-F. Brevet and H. H. Girault, Phys. Chem. Chem. Phys., 1999, 1, 1461-1467.

35 Z. Samec, N. Eugster, D. J. Fermin and H. H. Girault, J. Electroanal. Chem., 2005, 577, 323-337.

36 D. J. Fermin, H. Duong, Z. Ding, P.-F. Brevet and H. H. Girault, J. Am. Chem. Soc., 1999, 121, 10203-10210.

37 D. Schaming, M. Hojeij, N. Younan, H. Nagatani, H. J. Lee and H. H. Girault, Phys. Chem. Chem. Phys., 2011, 13, 17704-17711.

38 V. G. Levich, Physicochemical hydrodynamics, Prentice-Hall, 1962.

39 G. Zhao and M. Pumera, J. Phys. Chem. B, 2012, 116, 10960-10963.

40 A. G. Volkov, D. W. Deamer, D. L. Tanelian and V. S. Markin, Prog. Surf. Sci., 1996, 53, 1-134.

41 B. D. Fitchett, J. B. Rollins and J. C. Conboy, Langmuir, 2005, 21, 12179-12186.

42 R. A. Marcus, Angew. Chem., Int. Ed. Engl., 1993, 32, 1111-1121.

43 M. D. M. Scanlon, P. Peljo, M. A. Mendez, E. A. Smirnov, H. H. Girault, M. A. Méndez, E. A. Smirnov and H. H. Girault, Chem. Sci., 2015, 6, 2705-2720. 
44 E. Smirnov, P. Peljo, M. D. Scanlon and H. H. Girault, ACS Nano, 2015, 9, 65656575.

45 H. H. Girault and D. J. Schiffrin, in Electroanalytical Chemistry. A series of advances, ed. A. J. Bard, Marcel Dekker, New York, 1989, vol. 15, pp. 1-141.

46 T. Kakiuchi, J. Electroanal. Chem., 2002, 536, 63-69.

47 Y. Kitazumi and T. Kakiuchi, Langmuir, 2009, 25, 10829-10833.

48 L. Zhang, Y. Kitazumi and T. Kakiuchi, Langmuir, 2011, 27, 13037-13042.

49 N. M. Kovalchuk, Open Chem., 2015, 13, 1-16.

50 J. Turkevich, P. C. Stevenson and J. Hillie, Discuss. Faraday Soc., 1951, 11, 75-82.

51 G. Frens, Nature, 1973, 241, 20. 